\title{
USING DIFFERENT IODINE STAINING FOR MORPHOLOGICAL ANALYSIS FROM MICROCT IMAGES IN MUSCLE STRUCTURE OF WISTAR RATS
}

\author{
Fábio G. Piau *, Juliana Haddad, Ana C. Rossi, Felippe B. Prado, Alexandre R. Freire.
}

\section{Abstract}

The aim of this study was to evaluate the effect of concentration and staining duration of iodine contrast applied to study the masticatory muscle of Wistar rats in microCT images. The iodine contrast was applied with $3 \%, 5 \%$ and $10 \%$, during 7,15 and 30 days. The contrast with $10 \%$ presented the better results, in general..

\section{Key words:}

Masticatory muscles, computerized microtomography, contrast agents.

\section{Introduction}

Understanding masticatory biomechanics in Wistar rats is extremely important for better interpretation of studies in craniofacial biology applied to normal occlusion, occlusal changes and corrective occlusion treatments ${ }^{1}$. Therefore, it was necessary to perform an anatomical study of the masticatory muscles of this lineage.

As a general objective, this study evaluates the influence of the use of iodine-based contrast agents in the muscles of Wistar rats to characterize the 3D morphology based on computed microtomography images. Specifically, the objectives are to evaluate the best concentration, as well as the best fixation period, of the contrast to characterize the morphology of Wistar rat mastication muscles from computed microtomography images.

\section{Results and Discussion}

Twelve male Wistar rats (Rattus norvegicus albinus), 2 months old (200-250g) from CEMIB-UNICAMP were used. The animals were kept in collective cages (five animals/box), with a temperature of $22 \pm 2^{\circ} \mathrm{C}$, controlled light cycle (12/12 h) and free access to water and feed.

The animals were randomly assigned to the experiments in 3 groups of four rats each, with the iodine contrast solutions of $3 \%, 5 \%$ and $10 \%$. Each group was subdivided into 3 subgroups according to the staining duration: 7 days, 15 days and 30 days.

The pieces were scanned on a 1174 Skyscan microtomograph (Bruker, Belgium) with a voltage of $50 \mathrm{Kv}$ and an amperage of $800 \mu \mathrm{A}$ at the School of Dentistry of Piracicaba-UNICAMP. The scanning of the pieces was done with a pixel dimension equal to $31 \mu \mathrm{m}$. After scanning, the images were exported to the software NRecon Reconstruction (Bruker, Belgium) in which they were reconstructed three-dimensionally in axial sections presenting the $x$-ray attenuation coefficients with values relative to the muscle structure reinforced by contrast. The reconstructed images were exported to the software Materialise MIMICS v18 (Materialise, Belgium) to evaluate the anatomical characteristics as well as the density of masticatory muscles. These parameters were evaluated in each piece to compare the effects of different contrast concentration and staining duration.

Overall, the results showed that the solution with major concentration as well as a longer staining duration resulted in a more detailed anatomical features of the muscles.
The muscle fibers were possible to observe only in the $5 \%$ and $10 \%$. The pieces with major staining duration enhanced the muscle characteristics.

According to the grayscale density values, the solution with major concentration and staining duration presented high values.

Different experiments have been performed using different animal species and contrast solution protocols ${ }^{2}$ and both concentration and staining duration can influence the result. It is recommended to use lower concentrations and short staining duration for small animals. Cox and Jeffery $(2011)^{3}$ described an iodine staining protocol using a solution with $25 \%$ during 7 weeks in brown rat (Rattus norvegicus). These authors reported that the staining duration was not enough to stain the internal structures and an iodine injection was necessary. For Wistar rat, in this present study, the solution with $10 \%$ concentration presented the best result compared to lower concentrations, regardless the staining duration. Thus, the solution concentration was the most effective parameter to stain the muscle fibers, once the $3 \%$ solution in long staining duration presented worst results compared to the $10 \%$ solution in short staining duration.

\section{Conclusions}

Considering the limitations of this study, it can be concluded that the iodine stain solution with major concentration (10\%) presents better results compared to lower concentrations, even using lower concentration associated to long staining duration.

${ }^{1}$ Goto K.T.; Kajiya H.; Nemoto T.; Tsutsumi T.; Tsuzuki T.; Sato H. e Okabe K. Hyperocclusion stimulates osteoclastogenesis via CCL2 expression. J. Dent. Res. 2011, 90, 793-798.

${ }^{2}$ Gignac, P.M.; Kley, N.J.; Clarke, J.A.; Colbert, M.W.; Morhardt, A.C.; Cerio D., et al. Diffusible iodine-based contrast-enhanced computed tomography (diceCT): an emerging tool for rapid, high-resolution, 3-D imaging of metazoan soft tissues. J. Anat. 2016, 228, 889-909.

3 Cox, P.G. e Jeffery, N. Reviewing the morphology of the jaw-closing musculature in squirrels, rats, and guinea pigs with contrast-enhanced microCT. Anat. Rec. 2011, 294, 915-928. 